\title{
Bias in chemokine receptor signalling
}

\author{
Annelien J.M. Zweemer, Jimita Toraskar, Laura H. Heitman, and Adriaan P. IJzerman
}

Division of Medicinal Chemistry, Leiden Academic Centre for Drug Research, Leiden University, Einsteinweg 55, 2333 CC Leiden, The Netherlands

\begin{abstract}
Chemokine receptors are widely expressed on a variety of immune cells and play a crucial role in normal physiology as well as in inflammatory and infectious diseases. The existence of 23 chemokine receptors and 48 chemokine ligands guarantees a tight control and fine-tuning of the immune system. Here, we discuss the multiple regulatory mechanisms of chemokine signalling at a systemic, cellular, and molecular level. In particular, we focus on the impact of biased signalling at the receptor level; an emerging concept in molecular pharmacology. An improved understanding of these mechanisms may provide a framework for more effective drug discovery and development at a target class that is so relevant for immune function.
\end{abstract}

\section{Regulation of the chemokine system}

Chemokines are the most important regulators of leukocyte trafficking and play a central role in the immune system [1]. They act via abundantly expressed chemokine receptors, which belong to the family of $\mathrm{G}$ protein-coupled receptors (GPCRs) (Box 1), on a wide variety of immune cells. Activation of these chemokine receptors induces migration and differentiation of immune cells, which both are essential processes during innate and adaptive immune responses [2].

The chemokine-directed immune response involves a complex network of reactions that are carefully fine-tuned at multiple levels throughout the body (Figure 1). At the systems level this involves spatiotemporal and tissue-specific expression of chemokine receptors and their ligands. At the cellular level the chemokine receptor signal can be modulated by coexpression of many differentially expressed proteins on immune cells. Finally, there is growing evidence of biased signalling at the molecular level for chemokine receptors, which implies that different chemokine ligands activate different intracellular pathways although binding to the same receptor.

With regard to this bias at the receptor level, novel mechanistic insights have been attained lately due to the advances in X-ray crystallography and NMR methods to resolve the structure of membrane proteins, such as GPCRs. Several structures of chemokine receptors have been elucidated now, among which are chemokine CXC

Corresponding author: IJzerman, A.P. (ijzerman@lacdr.leidenuniv.nl).

Keywords: chemokines; chemokine receptors; biased signalling.

$1471-4906 / \$$ - see front matter

(c) 2014 Elsevier Ltd. All rights reserved. http://dx.doi.org/10.1016/j.it.2014.02.004 receptor (CXCR)1, CXCR4 and chemokine CC receptor (CCR)5 [3-5]. In addition, for the serotonin 5-hydroxytryptamine (HT) ${ }_{1 \mathrm{~B} / 2 \mathrm{~B}}$ and the $\beta_{2}$-adrenergic receptors a structural basis for biased signalling was reported [6,7]. Similar mechanisms for ligand bias are likely to be present for the family of chemokine receptors, because these are particularly prone to biased signalling due to the presence of multiple endogenous chemokine ligands.

So far there has only been limited success in clinical trials targeting chemokine receptors. We propose therefore to consider chemokine regulation and bias at multiple levels in order to better understand their intricacies. Thus, in this review we present a summary of chemokine receptor signalling at a systems, cellular, and molecular level. Immunologists should be aware of the bias that can be introduced at a molecular level, whereas pharmacologists need to keep in mind that their target molecule could be modulated or expressed differently at a systems level.

\section{Regulation of chemokine expression and receptor activation}

The human chemokine system consists of $\sim 23$ receptors and 48 ligands [IUPHAR/BPS Guide to Pharmacology, http:// www.guidetopharmacology.org, accessed on 07-02-2014], of which the classically signalling chemokine receptors are presented in Figure 2. Most chemokine receptors can be activated by multiple chemokines, and one chemokine often has the ability to activate multiple receptors. Although previously regarded as redundant, the unique expression patterns of the various chemokines suggest that they form the basis for a specific and fine-tuned functioning of the immune system [1]. This is not only important in normal physiology, but also during certain immunopathological disease states, as illustrated by the CCR2 receptor and its ligands. CCR2 can be activated by the chemokine ligands chemokine $\mathrm{CC}$ ligand (CCL)2/monocyte chemotactic protein (MCP)-1, CCL7/MCP3, CCL8/MCP-2, CCL11/eotaxin, CCL13/MCP-4, and CCL16/ human CC chemokine (HCC)-4. Most studies have been focused on the CCL2-CCR2 interaction because CCL2 is the endogenous ligand with the highest affinity for CCR2. Nevertheless, in infectious diseases, CCL7 has been found to be crucial for monocyte recruitment to inflammatory sites mediated through CCR2 [8]. An example of distinct expression patterns observed in immunopathology is the regulation of the CCR4 ligands CCL17/thymus- and activationregulated chemokine (TARC) and CCL22/macrophagederived chemokine (MDC), which are not expressed in healthy skin tissue [9]. However, in inflamed skin lesions, CCL17 is detected on endothelial cells, whereas CCL22 is only presented by dendritic cells [9]. This distinct chemokine 


\section{Box 1. Chemokine receptors as GPCRs}

GPCRs

- With $>800$ members, GPCRs are the largest family and most diverse group of cell surface receptors and the most common target for therapeutic drugs [79].

- The GPCR structure consists of an extracellular $\mathrm{N}$ terminus, an intracellular $C$ terminus, and seven transmembrane (TM) helices, connected by three cytoplasmic and three extracellular loops [80].

- Ligand binding mostly takes place in a pocket formed by the seven helices close to the extracellular side of the receptor; it induces a conformational change at the intracellular side of the receptor that results in receptor activation and subsequent signalling [81].

- At the intracellular side different effector proteins can bind and transduce signals, among which are $G$ proteins and $\beta$-arrestins [82].

Chemokine receptors

- Chemokine receptors belong to the class A rhodopsin-like family of GPCRs.

- 23 different chemokine receptors have been identified that can be activated by $\sim 48$ chemokine ligands [IUPHAR/BPS Guide to Pharmacology, http://www.guidetopharmacology.org, accessed on 07-02-2014].

- Four subclasses of chemokine ligands have been identified on the basis of the pattern of conserved cysteine residues $(C, C C, C X C$, and $\left.\mathrm{CX}_{3} \mathrm{C}\right)$ [83].

- Chemokine receptors have been classified as C, CC, CXC, and $\mathrm{CX}_{3} \mathrm{C}$ receptors based on the chemokine subclass ligand that they bind.

- Most chemokine receptors bind multiple chemokines, and most chemokines can bind to and activate multiple chemokine receptors.

- The chemokine receptors ACKR1 (DARC), ACKR2 (D6), ACKR3 (CXCR7), and ACKR4 (CCX-CKR) are so-called decoy receptors that predominantly scavenge chemokine ligands from the extracellular environment, although some of these also couple to $\beta$-arrestins [16].

expression pattern has been demonstrated in diseases ranging from psoriasis to atopic dermatitis, therefore, this could be a general feature underlying the disease state. In general the balance, timing, and pattern of chemokine expression

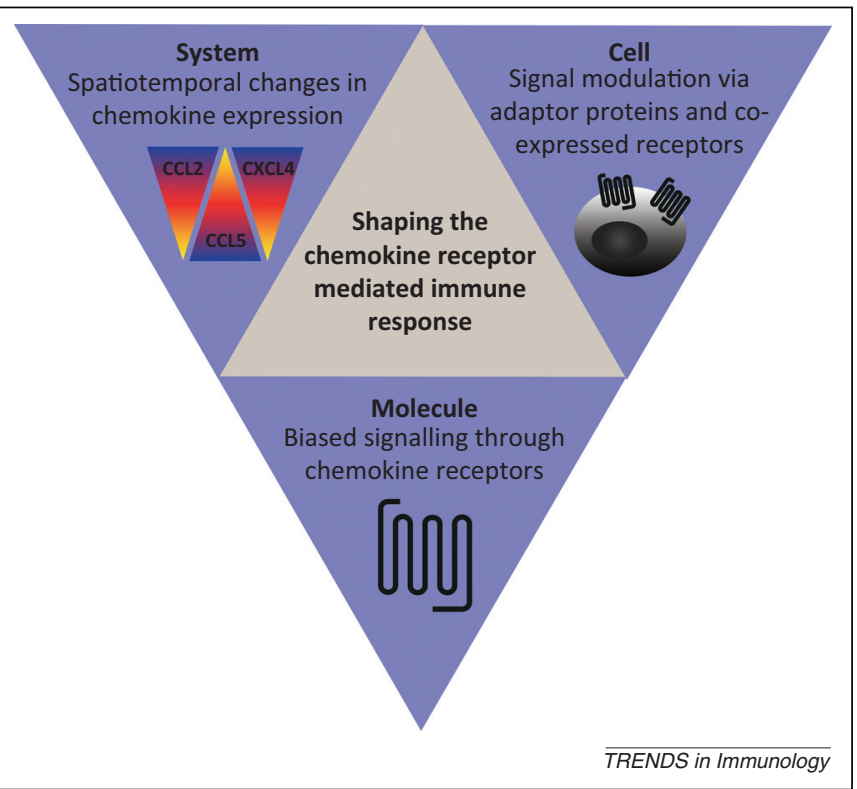

Figure 1. Schematic representation of the structure of this review. The chemokine receptor-mediated immune response is discussed at a systems, cellular, and molecular level. appears to regulate the generation of immune-cell-specific responses in health and disease [10].

In addition to the difference in release and production of chemokines among various tissues, their in vivo availability also depends on the interaction of chemokines with specific glycosaminoglycan (GAG) chains that are presented at the cell surface as part of membrane proteoglycans. The binding of chemokines to GAGs allows immobilization, accumulation, and retention of chemokines on cell surfaces near their sites of production in order to provide directional signals to migrating cells [11]. In addition, GAG interactions are involved in the transport of chemokines across cell surfaces. GAGs may selectively bind chemokines and therefore fine-tune the immune response, because they display varying affinities for specific chemokines and are differentially expressed in time and location on specific cell types and tissues [12]. Furthermore, cells and tissues can alter the expression of GAGs in pathophysiology. This has been observed upon inflammatory stimuli in diseases of the gastrointestinal tract as well as in multiple different tumours $[13,14]$. GAGs might even be directly involved in signalling, because their attached core proteins that span the membrane can undergo tyrosine phosphorylation and thereby contribute to signal transduction, as reported for CXCL12/SDF-1 and the proteoglycan syndecan-4 [15]. Although they are a crucial factor for chemokine signalling, the exact functional consequences of chemokine-GAG interactions and the level of specificity are still largely speculative.

Not only GAGs can alter the availability of chemokines, but also chemokine receptors themselves. A certain group of chemokine receptors, known as atypical chemokine receptors (ACKRs) [16], have been proposed to act mainly as chemokine ligand scavengers $[17,18]$. Furthermore, under certain circumstances the G protein-coupled chemokine receptors have been demonstrated to become uncoupled from $\mathrm{G}$ protein signalling. For example, dendritic cells and monocytes treated with anti-inflammatory interleukin (IL)-10 express 'uncoupled' or 'nonsignalling' CCR1, CCR2, and CCR5, which can scavenge their corresponding inflammatory chemokines in vitro as well as in mice [19]. Another study demonstrated both in vitro and in vivo that apoptotic leukocytes express 'silent' CCR5 receptors, scavenging CCR5 ligands, and thereby contributing to the resolution of inflammation in a mouse model of peritonitis [20]. Therefore, expression of a certain chemokine receptor does not always imply a contribution to the disease state. In fact, one might speculate that a pharmacological blockade of these receptors can increase free chemokine levels and therefore result in enhanced pathology.

Altogether, the examples above illustrate that the expression of chemokines and their receptors varies over time and between different conditions, and studies of mechanisms and outcomes associated with this differential expression in several disease states have been reviewed previously [21,22]. As noted above, it is clear that expression of chemokines and their receptors does not necessarily imply a role as stimulator or enhancer of a pathophysiological state, which is an important factor to consider while developing antagonists targeting the chemokine system. 


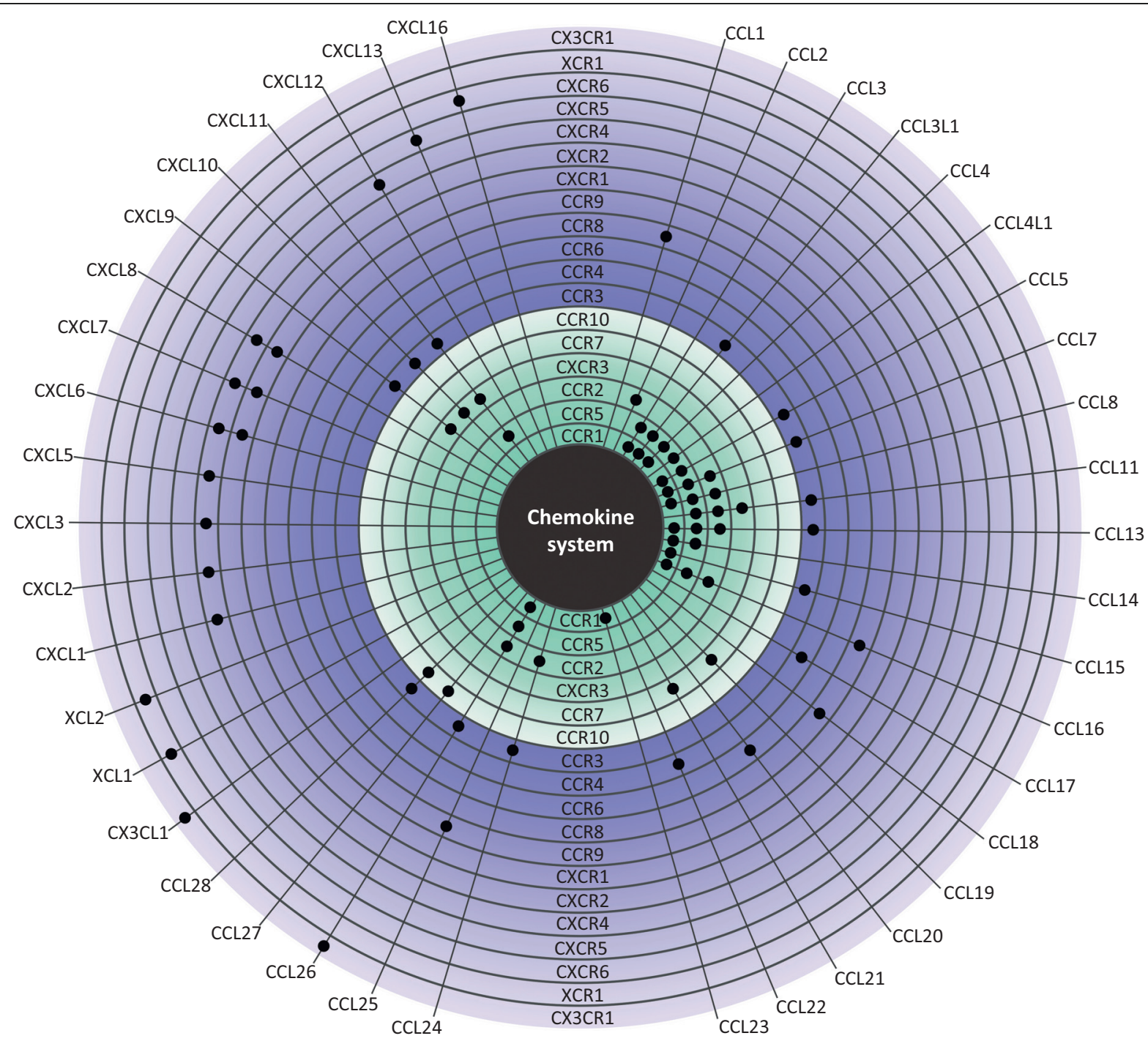

TRENDS in Immunology

Figure 2. Overview of the family of chemokines and chemokine receptors. The green inner circle represents those chemokine receptors for which some form of biased signalling has been documented. This is not (yet) the case for the chemokine receptors in the blue outer circle. The black dots represent the chemokine ligands that have been shown to bind to a given chemokine receptor. The group of atypical chemokine receptors (ACKRs) is not depicted in this figure.

Besides the regulation of chemokines and their receptors throughout the body, there is substantial evidence that chemokine receptors modulate each other within a particular immune cell. This is discussed in the following section.

\section{Regulation of chemokine receptor signalling in immune cells}

Chemokine receptors are expressed by immune cells in both the innate and adaptive compartments, including $\mathrm{B}$ and $\mathrm{T}$ lymphocytes, monocytes, and neutrophils [23]. Distinct expression profiles characterize the different leukocyte subtypes. For example, in T helper (Th) cells, several chemokine receptors are associated with the Th1 phenotype (including CXCR3 and CCR5), whereas others are associated with the Th2 phenotype (including CCR4 and CCR8). This phenomenon is likely related to their discriminate functions in response to viral and bacterial pathogens or during allergic reactions [24,25]. In the case of monocytes, a different repertoire of chemokine receptors can be expressed depending on environmental factors and stimuli. Lipopolysaccharide (LPS) downregulates CCR1, CCR2, and CCR5 expression in monocytes, whereas IL-2 stimulates CCR2 expression [26]. In addition, CCR7 is upregulated upon immunogenic stimulation, possibly to facilitate lymph-node homing $[27,28]$. Tight regulation of the different chemokine receptors on immune cells therefore shapes the immune cell response.

The majority of immune cells express multiple chemokine receptors simultaneously. At a cellular level, chemokines can counteract each other or display synergy, thereby reducing the inflammatory response or increasing the selectivity of cell recruitment $[29,30]$. For example, via heterologous receptor desensitization or internalization one chemokine can lower the responsiveness of a cell to other chemokines binding to a distinct chemokine receptor [31]. This phenomenon has been studied in human peripheral blood T cells, which express CCR5 and CXCR4 [32]. Upon simultaneous addition of their chemokines CCL4/ macrophage inflammatory protein (MIP)-1 $\beta$, CCL5/ regulated on activation, normal $\mathrm{T}$ cell expressed and 
secreted (RANTES), and CXCL12, the capacity of CXCL12 to induce chemotaxis in vitro is suppressed. This crosstalk does not involve the internalization of the receptor, but rather a cross-desensitization via a decrease in phosphorylation of downstream signalling proteins. The rich chemokine environment surrounding the leukocytes during inflammatory conditions can therefore induce different cellular responses than determined in assays that only reflect the behaviour of one particular chemokine receptor and ligand [33].

Chemokines can also modulate signalling responses through other chemokine receptors due to the presence of heterodimeric or hetero-oligomeric receptor complexes [34]. This has been demonstrated for several chemokine receptors, among which CCR2, CCR5, and CXCR4 [35-37]. In CCR2-CCR5 heterodimers, the CCR5 ligands CCL3/ MIP-1 $\alpha$, CCL4, and CCL5 were able to displace CCL2 from CCR2 [37]. This so-called negative cooperativity was further analysed in different in vitro assays to confirm the allosteric nature of this displacement via heterodimers [36]. The relevance for immune cell functioning has been demonstrated as well, because negative binding cooperativity takes place in hetero-oligomeric complexes between the binding pockets of CCR2, CCR5, and CXCR4 in T cells and monocytes that endogenously express these receptors [35]. As a result, the recruitment of these cells mediated by the CXCR4 agonist CXCL12 in mice could be inhibited by antagonists of CCR2 and CCR5.

Within immune cells, the magnitude and duration of the signal depends on the exposed chemokine concentration and on (subsequent) chemokine receptor desensitization, phosphorylation, and internalization. These processes are regulated via G protein-coupled receptor kinases (GRKs) and $\beta$-arrestins [38]. On the intracellular side of the cell, the different repertoires of these adaptor proteins regulate the eventual cellular effects. In RBL-2H3 cells stably expressing both receptors, it has been shown that CXCR1 and CXCR2 couple to distinct GRK isoforms [39]. CXCR1 predominantly couples to GRK2, whereas CXCR2 interacts with GRK6 to negatively regulate receptor sensitization and trafficking, eventually affecting cell signalling [39,40]. The role of GRK6 in neutrophil recruitment was further demonstrated in studies using wild type and GRK6 ${ }^{-1-}$ knockout mice [39]. In addition, different types of immune cells express different types of GRKs and $\beta$-arrestins; the levels of which may also vary, adding another layer of bias and fine-tuning of the response of chemokines and their receptors $[41,42]$.

Thus, there are multiple co-receptors and adaptor proteins that define the eventual chemokine receptor signal. In order to study the effect of a chemokine or potential drug candidate, it is important to include cell types and tissues that reflect the in vivo situation more so than cell lines, devoid of physiological context, with heterologous receptor expression.

\section{Biased signalling through chemokine receptors}

At the molecular level yet another type of bias is present in the chemokine system, because chemokine receptors are capable of differentially signalling in a ligand-specific manner. This biased signalling, also called functional selectivity, refers to agonist ligands that favour the activation of a certain intracellular signalling pathway over another [43]. The following sections discuss the multiple intracellular signalling routes that can be activated by chemokines. The aim is to give a comprehensive overview of the biased signalling events that have been reported for chemokine receptors so far, illustrating that the chemokine system is extensively fine-tuned at the receptor level already.

\section{GPCR signalling}

GPCRs transduce the effects of many extracellular signals/ ligands (whether those are chemokines or other hormones and neurotransmitters) to intracellular pathways and signalling routes (Figure 3 ). They bind to and activate heterotrimeric $G$ proteins that consist of $\mathrm{G}_{\alpha}, \mathrm{G}_{\beta}$, and $\mathrm{G}_{\gamma}$ subunit, for which 21,6 , and 12 different types are present in humans, respectively [44]. Activation of these G proteins modulates the production of second messenger molecules such as cyclic AMP (cAMP), intracellular calcium $\left(\mathrm{Ca}^{2+}\right)$, and inositol phosphates (IPs), which control further downstream effectors such as protein kinase (PK)C and Akt. GPCR activation and consequently G protein-mediated signalling are terminated via phosphorylation of the GPCR by GRKs. The phosphorylated receptor recruits $\beta$-arrestins, of which various subtypes exist. This association often results eventually in receptor internalization to the cytosol, effectively impeding further signalling from the receptor. After receptor coupling, $\beta$-arrestins are also able to transduce signals themselves, for example, via subsequent activation of the extracellular signal-regulated kinase (ERK) pathway [45]. For the purpose of the present discussion, we focus on the major signalling pathways via $G$ proteins and $\beta$-arrestins to illustrate the phenomenon of biased signalling through chemokine receptors in functions of the immune system.

In case of extreme signal bias through GPCRs, one ligand may mainly activate $G$ proteins, whereas another ligand only activates $\beta$-arrestins. This results in different cellular effects ('texture') although both ligands act via the same receptor; a process that has been extensively studied and discussed for GPCRs in general, as reviewed by Kenakin and Christopoulos [46]. Biased signalling does not only comprise distinct signalling via either $\mathrm{G}$ proteins or $\beta$ arrestins, but also includes more subtle differences in the activation of other downstream signalling proteins. For example, ligands can discriminate between different types of G proteins, whereas others differently affect signalling events such as ERK activation or $\mathrm{Ca}^{2+}$ mobilization. It is important to note that pathway activation depends also on the expression level of the receptor as well as the cellular expression and availability of signalling molecules, which result in cell-specific differences (Figure 3).

Advances in structural biology have led to an accumulating understanding of the underlying mechanisms. The first structural features in a GPCR crystal structure that are responsible for biased signalling were recently revealed for the serotonin receptors $5-\mathrm{HT}_{1 \mathrm{~B}}$ and $5-\mathrm{HT}_{2 \mathrm{~B}}$ [6]. Conformational changes at the intracellular side in their helix VI and helix VII were reported to be responsible 


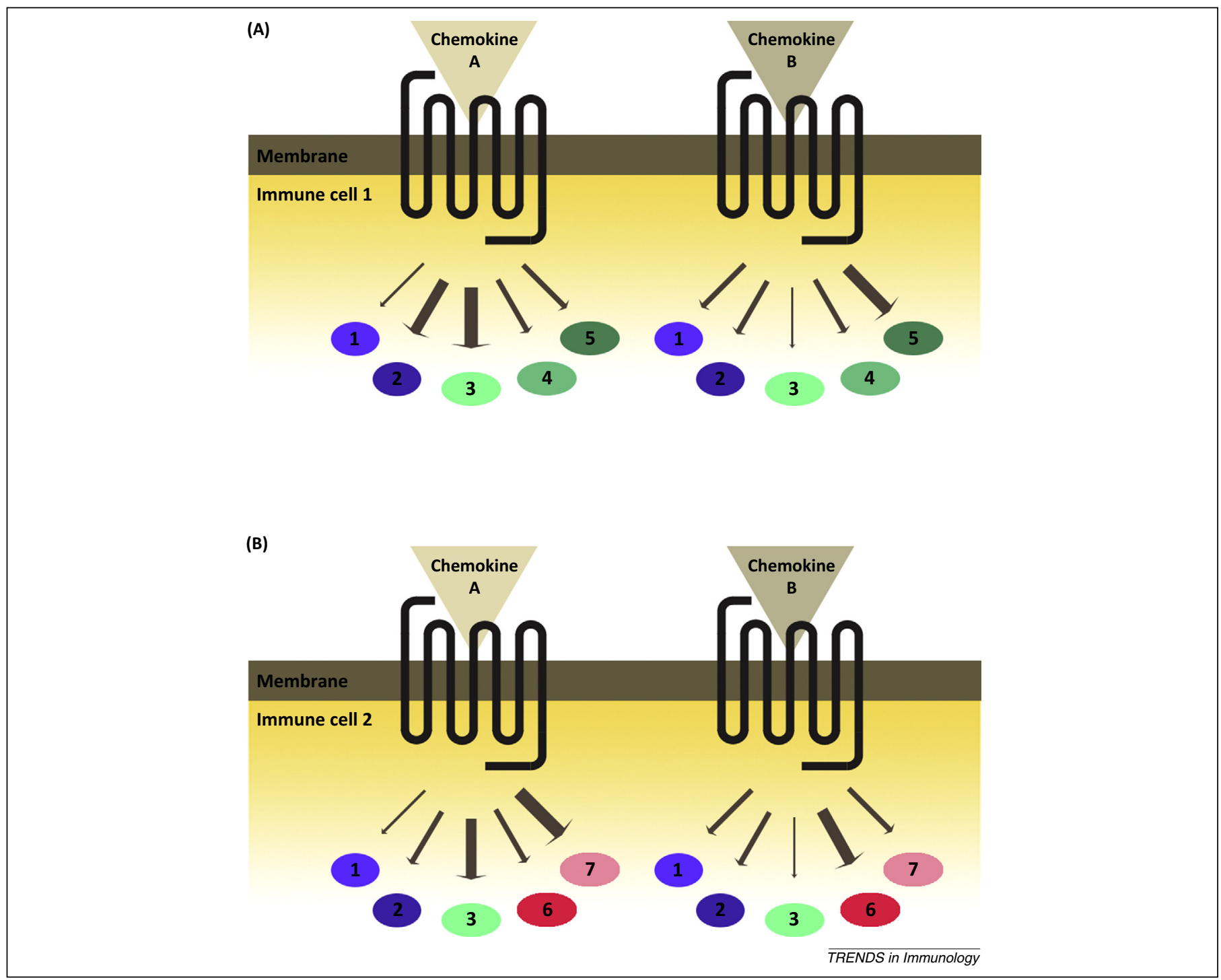

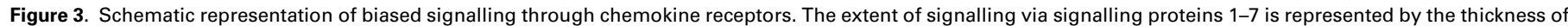

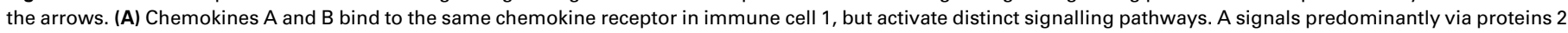
and 3 , whereas B signals mainly via protein 5 . (B) Immune cell 2 expresses signalling proteins different from immune cell 1 , which results in differential signalling profiles for chemokine $\mathrm{A}$ and $\mathrm{B}$.

for G protein signalling or $\beta$-arrestin signalling, respectively $[6,47,48]$. In addition to the 'snapshots' of bias in crystal structures, the emerging field of protein molecular dynamics further contributes to our understanding of ligand bias. Such studies have simulated at the atomic level how small perturbations at the more extracellularly located ligand binding site can lead to large conformational changes at the intracellular side of the receptor [49]. Importantly, not only do we start to understand the molecular features of biased signalling, we now also recognize its implications as it may lead to the development of therapeutics that have selective efficacy and fewer side effects [50].

The chemokine receptor family is prone to ligand-induced biased signalling, because most chemokine receptors can be activated by multiple chemokines. Interestingly, this is different from many other GPCRs with only one endogenous ligand, where ligand bias has largely been observed for synthetic agonists only. In several studies the activation of multiple pathways has been compared among the different chemokines, and for certain chemokine receptors, a biased signalling pattern has been discovered (Figures 2 and 3). These receptors include CCR1 [51-54], CCR2 [55-57], CCR5 [58-60], CCR7 [61-63], CCR10 [52], and CXCR3 [52,64]. With the exception of the homeostatic chemokine receptor CCR7, all are inflammatory chemokine receptors that bind multiple chemokines. The following sections discuss the biased signalling through each of these receptors individually.

\section{Biased signalling through CC chemokine receptors}

CCR1. The expression of CCR1 is known to coincide with the $\mathrm{G}$ protein subtypes $\mathrm{G}_{\mathrm{i} / \mathrm{o}}, \mathrm{G}_{14}$ and $\mathrm{G}_{16}$ [65]. Four chemokines, CCL3, CCL5, CCL7, and CCL15/HCC-2, were found to differentially activate these $\mathrm{G}$ protein subtypes in COS-7 cells or HEK293 cells transfected with CCR1, resulting in different intracellular levels of cAMP, ERK, and IP [51]. More specifically, CCL15 was discovered to be the only chemokine ligand that did not signal via $\mathrm{G}_{14}$ and $\mathrm{G}_{16}$. The inability of CCL15 to activate CCR1 as effectively 
as the others could potentially be caused by its long $\mathrm{N}$ terminal region. The $\mathrm{N}$-terminal region is responsible for receptor activation, whereas the $\mathrm{N}$-loop region is responsible for receptor binding. CCL15 can bind the receptor with high affinity due to its structural homology to other CC chemokines in the $\mathrm{N}$-loop region, but its extended $\mathrm{N}$-terminal region largely precludes subsequent receptor activation [53]. Further support comes from the finding that neither CCL14/HCC-1 nor CCL23/myeloid progenitor inhibitory factor (MPIF)-1, both having a long N-terminal region, can activate CCR1 despite sufficient binding affinity [51]. Another study on CCR1 agonism examined a total of eight different chemokine ligands, and reported that CCL8 was a poor G protein activator, whereas CCL4 was proposed to act as an in vivo inhibitor of CCR1 in the leukaemia cell line HL-60 because it showed only marginal receptor activation in functional assays [54]. In CCR1transfected HEK293 cells, CCL5 and CCL23 have been identified as G protein-biased chemokines compared to CCL3, whereas CCL5, CCL15, and CCL23 display bias for internalization following $\beta$-arrestin recruitment [52]. All these different studies illustrate that CCR1 is heavily prone to biased signalling.

CCR2. The CCR2 receptor has been reported to bind eight chemokines, namely CCL2, CCL7, CCL8, CCL11, CCL13, CCL16, CCL24/eotaxin-2, and CCL26/eotaxin-3. Berchiche et al. studied the $\mathrm{G}_{\mathrm{i}}$ activation and $\beta$-arrestin recruitment profiles of these different ligands in HEK293 cells transfected with CCR2 [55]. Overall, the potency and efficacy rank orders of $\mathrm{G}$ protein activation and $\beta$-arrestin recruitment were comparable. However, when the $\beta$-arrestin subtypes 1 and 2 were compared, the weak arrestin recruiters CCL8 and CCL13 were found to show bias towards $\beta$ arrestin 2 . Interestingly, the study also reported the kinetics of the CCR2- $\beta$-arrestin complexes. CCL7 had a high potency to recruit $\beta$-arrestin, but with a short half-life of the signal, whereas CCL8 and CCL13 induced weak but stable signals over time. These differences in kinetic patterns are additional factors leading to signal bias that deserve more attention; it has been reported for other GPCRs that this can be an underlying factor for differential signalling as well as signal termination [66]. Besides the signalling properties of the different CCR2 ligands, it has been reported that CCL2, CCL7, and CCL13 induce homodimer formation of CCR2, whereas the other chemokines do not [55]. The formation of dimers, hetero- or homo-, is yet another mechanism to create bias in signalling and should therefore be taken into account when interpreting signal bias of any chemokine receptor.

CCR5. The signalling properties of CCL3, CCL4, CCL5, CCL8, and CCL13 via CCR5 have been investigated in cAMP accumulation and $\mathrm{Ca}^{2+}$ mobilisation assays in $\mathrm{CHO}$ cells stably expressing CCR5. The potency rank order of chemokine ligands was similar between the two assays, however, CCL13 was completely unable to affect cAMP concentrations [58]. The $\mathrm{Ca}^{2+}$ responses were $\mathrm{G}$ protein dependent, except for CCL3 that produced $\mathrm{a} \mathrm{Ca}^{2+}$ signal in a partly $\mathrm{G}$ protein-independent manner. These results illustrate that the CCR5 chemokines act differently.
Interestingly, other groups that used different cell systems, including RBL-2H3 and COS-7 cells expressing CCR5, reported different rank orders of signalling efficiencies $[60,67]$. This indicates cell-type specific effects or differential activation of the various intracellular effectors by these chemokines (Figure 3). It further emphasizes that the choice of cell lines must be an important parameter; primary cell lines and immune cells may better represent an in vivo relevant bias in signalling. The underlying mechanism for the differential effects of CCR5 ligands has been addressed in studies that focussed on the structural determinants at the receptor level. Biased signalling could be a result of ligand-specific induction of phosphorylation sites and thereby specific recruitment of GRKs, which differs among the CCR5 chemokine ligands [60]. A mutagenesis study further explored molecular determinants of CCR5-induced signalling. Residues in helices VI and VII were identified that are responsible for causing biased signalling [59]. In the crystal structures of the serotonin receptors it was the orientation of these same helices that was found to induce biased signalling [6]. It would be interesting to determine if these structural features can be translated to other chemokine receptors as well, which would be helpful for drug development targeting these receptors.

CCR7. CCL19/Epstein-Barr virus-induced molecule 1 ligand chemokine (ELC) and CCL21/secondary lymphoid tissue chemokine (SLC) are the endogenous agonists for CCR7. They are equally active in promoting $G$ protein activation, $\mathrm{Ca}^{2+}$ mobilization, and chemotaxis [62,63]. However, in terms of $\beta$-arrestin recruitment and subsequent internalization of the receptor they behave differently. It was found that $\beta$-arrestin 3 was responsible for CCR7 internalization in the $\mathrm{T}$ cell lymphoma cell line HuT78 after stimulation with CCL19, which was not the case for CCL21-induced internalization [61]. Although both ligands allowed recruitment of $\beta$-arrestin 2 , only CCL19 led to redistribution of $\beta$-arrestin-2 into endocytotic vesicles and classical receptor desensitization in HEK293 cells stably expressing CCR7 [62]. The underlying mechanism for this difference in $\beta$-arrestin recruitment was found to be a differential recruitment of GRKs that phosphorylate CCR7. CCL19 activates both GRK3 and GRK6, whereas CCL21 activates GRK6 only. Kohout and co-workers have reported findings that support this differential recruitment of GRKs, revealing that CCL19 induces specific phosphorylation sites on CCR7 in HEK293 cells [63].

CCR10. CCL27/cutaneous $\mathrm{T}$ cell-attracting chemokine (CTACK) and CCL28/mucosae-associated epithelial chemokine (MEC) are the two endogenous chemokines for CCR10. Both ligands are capable of $\mathrm{G}$ protein signalling measured by cAMP accumulation, but only CCL27 is capable of recruiting $\beta$-arrestin and internalizing the receptor [52]. In the same study it was assessed that CCL28 displayed a higher efficacy for migration of peripheral blood mononuclear cells (PBMCs). The biased signalling of CCL28 was speculated to be causal for this effect, explained by the lack of $\beta$-arrestin signalling and internalization that could result in prolonged and unopposed G protein activation [52]. 
Biased signalling through CXC chemokine receptors and ACKRs

CXCR3. CXCR1, CXCR2, and CXCR3 are the three CXC chemokine receptors that can each bind to multiple chemokines, but thus far biased signalling has only been reported for CXCR3. The activation of CXCR3 by CXCL9/monokine induced by gamma-interferon (MIG), CXCL10/IP-10 and CXCL11/interferon-inducible $\mathrm{T}$ cell alpha chemoattractant (I-TAC) has been investigated in a label-free impedancebased cellular assay [64]. For this technique, low-voltage currents run through microelectrode sensors at the bottom of a plate to which cells are attached. Changes in impedance upon ligand addition are continuously measured, and reflect receptor activation in the absence of a chemical or biological biosensor within the cell. Using this technique, Watts et al. showed that CXCL9 behaved as a biased CXCR3 agonist in HEK293 cells stably expressing CXCR3, stimulating solely G protein-dependent pathways [64]. However, another study compared cAMP accumulation, $\beta$-arrestin recruitment and internalization in classical functional assays with HEK293 cells transfected with CXCR3, and reported the opposite finding of CXCL9 being $\beta$-arrestin biased [52]. In addition CXCL11 was biased towards internalization. Apparently, CXCR3 is subject to biased signalling, of which the extent and nature may be determined by the different receptor expression levels and cellular assays used (Figure 3). Although future studies will ascertain whether biased signalling through other CXC receptors exists, we would hypothesize that this is likely, because, for example, CXCR2 binds seven different chemokine ligands.

ACKRs. It should be noted that the ACKRs [16] such as ACKR1 (Duffy antigen/receptor for chemokines (DARC)), ACKR2 (D6), ACKR3 (CXCR7), and ACKR4 (chemocentryx chemokine receptor (CCX-CKR)) can bind a wide variety of chemokine ligands, with up to 18 ligands for ACKR1. These receptors were initially regarded as nonsignalling decoy receptors because of their inability to activate typical G protein-signalling pathways, and therefore ACKRs were proposed to only scavenge ligands or function as co-receptors [17]. However, some recent studies have shown that these ACKRs can signal predominantly through $\beta$-arrestins. The chemokines CXCL11 and CXCL12 induce $\beta$ arrestin-2 recruitment to ACKR3 transfected in CHO cells, and a small molecule ACKR3 ligand has been reported to induce this $\beta$-arrestin-2 recruitment with even higher potency and efficacy [68]. Another study has also reported that CXCL12 induces $\beta$-arrestin-2 recruitment to ACKR3 in HEK293 cells transfected with ACKR3, as well as rat vascular smooth muscle cells (VSMCs), which results in downstream activation of ERK [69]. These signalling properties were discovered to be important in physiologically relevant cell lines, because the ACKR3-mediated migration of rat VSMCs was found to be a $\beta$-arrestin-mediated process [69]. Besides ACKR3, ACKR2 can also recruit $\beta$ arrestins, however, further evidence of subsequent signalling remains to be elucidated for this receptor [70]. Given these reports of $\beta$-arrestin recruitment and signalling through ACKRs, it has been proposed that these receptors should be regarded as a subfamily of $\beta$-arrestin-biased GPCRs. However, there is now one case reported of ACKR3 signalling via $\mathrm{G}_{\mathrm{i} / \mathrm{o}}$ in primary rodent astrocytes and human glioma cells [71]. This finding reveals the presence of thus far hidden properties of ACKRs, and the exact role and significance of ACKR-mediated signalling remains an open question for future research. ACKRs serve homeostatic functions by clearing chemokines from circulation and tissues [72], and in addition viruses and parasites are capable of modulating the expression of ACKRs to elude chemokines [73]. These receptors are therefore important for innate and adaptive immunity, emphasizing the necessity to resolve their functioning.

\section{Concluding remarks}

Here, we have discussed the regulation and bias in chemokine expression and signalling at a systems, cellular, and molecular level. The presented evidence implies that the focus on only one natural ligand of a chemokine receptor as a prototype agonist is insufficient; neither should we focus on one single chemokine receptor in vitro because there might be several other receptors that determine the fate of the immune cell in vivo.

Over the past few years the 'drugability' of chemokine receptors has been reviewed and questioned [74]. The family of chemokine receptors is involved in a wide variety of diseases, mostly characterized by chronic inflammation.

\section{Box 2. Chemokine signalling: implications for drug discovery in immunology}

The complexity of the chemokine receptor family and its ligands should be considered during drug discovery. Most chemokine receptors are activated by multiple chemokine ligands, which should be taken into account upon developing small molecule antagonists. This can be illustrated by research on the chemokine receptor CCR1. For this receptor, it has been shown that small molecule ligands can both act as allosteric enhancers for CCL3 and at the same time as a competitive blockers of the binding of CCL5 [84]. This drug-induced bias is important, because chemokines are differentially expressed and regulated during immunopathology.

Not only small molecule ligands, but also short lipidated peptide sequences named pepducins have been found to be candidate drugs targeting chemokine receptors. The pepducin ATI-2341 selectively targets CXCR4 and is an allosteric agonist in vitro as well as in vivo [85]. In a recent study it was discovered that ATI-2341 revealed functional selectivity for $G_{i}$ pathways over $G_{13}$ and $\beta$ arrestin [86]. In comparison to the CXCR4 antagonist AMD-3100, which is used in the clinic to mobilize haematopoietic stem cells from the bone marrow for transplantation of leukaemia patients [76], the pepducin ATI-2341 does not induce the additional undesired mobilization of lymphocytes [85]. Whether CXCR4mediated biased signalling of ATI-2341 with respect to AMD-3100 is causal for this difference remains a question for further research.

Besides the pepducins, small molecule ligands can also induce biased signalling upon binding to chemokine receptors $[59,64,87,88]$. For CCR5, the small molecule agonists YM-370749 and $E S N-196$ are able to induce $\mathrm{G}$ protein coupling and activation of $\mathrm{Ca}^{2+}$ responses $[87,88]$. However, they do not stimulate chemotactic activity but instead induce internalization of CCR5 from the cell surface $[87,88]$. These ligands are therefore functionally selective CCR5 ligands that act differently from the chemokine CCL5. This biased profile of small molecules acting via CCR5 could be of use as a novel class of anti-HIV-1 therapeutics, for which internalization of the receptor has been found to inhibit viral entry to the cell.

In conclusion, it is important to consider that drugs might differently affect one chemokine receptor depending on the chemokine that binds this receptor. In addition, these drugs can exert biased signalling via chemokine receptors themselves. 
Although $>50 \%$ of these receptors have been examined in clinical trials in the past decade, only two drugs targeting chemokine receptors have been approved by the regulatory authorities. The CCR5 antagonist maraviroc inhibits entry of HIV into CCR5-positive cells [75], and the CXCR4 antagonist AMD3100 is used to mobilize human haematopoietic stem cells from the bone marrow [76]. Notably, neither of these conditions is an inflammatory disease, nor is biased signalling involved. Possible reasons for the failure of other drug candidates targeting chemokine receptors are: (i) lack of efficacy due to inappropriate disease models; (ii) lack of efficacy due to poor pharmacokinetics such as binding to serum components; and (iii) offtarget (side) effects. The complexity at multiple levels of the chemokine system is another factor that introduces challenges for effective intervention in disease states (Box 2). Integration of these issues in early stages of drug discovery and development programmes is necessary in order to develop clinically effective drugs with an acceptable benefit/risk profile. The clinical relevance of biased ligands is illustrated by the $\mu$-opioid receptor agonist TRV130, which has recently been tested in Phase I studies and is entering Phase II in 2014 for the treatment of acute pain [77]. TRV130 causes G protein signalling with similar potency and efficacy as morphine, but with far less $\beta$-arrestin recruitment and receptor internalization [78]. Subsequent studies in mice have demonstrated that this bias results in higher analgesic efficacy, less gastrointestinal dysfunction, and less respiratory suppression than morphine.

In summary we have reviewed the regulation of and bias in chemokine receptor signalling that should be taken into account by immunologists as well as pharmacologists (Figure 1). The expression profile of chemokine ligands at the target tissue should be determined and considered in order to design appropriate in vitro studies, taking into account the differential expression profiles in health and disease. This is important given the aforementioned data that have reported crucial and different roles between chemokine ligands that bind to one chemokine receptor. In addition, the growing evidence of biased signalling through chemokine receptors shows that we cannot restrict ourselves to incorporate one chemokine ligand only, because the effect of drug candidates in the presence of other chemokine ligands might be different. Although heterologous cell systems can be used at the start of a project, the function of the chemokine receptor and impact of a drug should be studied in physiologically relevant cells as early as possible, because coexpressed proteins and receptors can modulate the behaviour and response of a chemokine receptor. It is hoped that this review will help in designing such experiments.

\section{References}

1 Zlotnik, A. and Yoshie, O. (2012) The chemokine superfamily revisited. Immunity 36, 705-716

2 Esche, C. et al. (2005) Chemokines: key players in innate and adaptive immunity. J. Invest. Dermatol. 125, 615-628

$3 \mathrm{Wu}$, B. et al. (2010) Structures of the CXCR4 chemokine GPCR with small-molecule and cyclic peptide antagonists. Science 330, 1066-1071

4 Tan, Q. et al. (2013) Structure of the CCR5 chemokine receptor-HIV entry inhibitor maraviroc complex. Science 341, 1387-1390
5 Park, S.H. et al. (2012) Structure of the chemokine receptor CXCR1 in phospholipid bilayers. Nature 491, 779-783

6 Wacker, D. et al. (2013) Structural features for functional selectivity at serotonin receptors. Science 340, 615-619

7 Liu, J.J. et al. (2012) Biased signaling pathways in beta2-adrenergic receptor characterized by 19 F-NMR. Science $335,1106-1110$

8 Tsou, C.L. et al. (2007) Critical roles for CCR2 and MCP-3 in monocyte mobilization from bone marrow and recruitment to inflammatory sites. J. Clin. Invest. 117, 902-909

9 D'Ambrosio, D. et al. (2002) Quantitative differences in chemokine receptor engagement generate diversity in integrin-dependent lymphocyte adhesion. J. Immunol. 169, 2303-2312

10 Groom, J.R. and Luster, A.D. (2011) CXCR3 ligands: redundant, collaborative and antagonistic functions. Immunol. Cell Biol. 89, 207-215

11 Tanino, Y. et al. (2010) Kinetics of chemokine-glycosaminoglycan interactions control neutrophil migration into the airspaces of the lungs. J. Immunol. 184, 2677-2685

12 Lortat-Jacob, H. (2009) The molecular basis and functional implications of chemokine interactions with heparan sulphate. Curr. Opin. Struct. Biol. 19, 543-548

13 Sasisekharan, R. et al. (2002) Roles of heparan-sulphate glycosaminoglycans in cancer. Nat. Rev. Cancer 2, 521-528

14 Gotte, M. (2003) Syndecans in inflammation. FASEB J. 17, 575-591

15 Charnaux, N. et al. (2005) Syndecan-4 is a signaling molecule for stromal cell-derived factor-1 (SDF-1)/CXCL12. FEBS J. 272, 1937-1951

16 Bachelerie, F. et al. (2013) International Union of Pharmacology. LXXXIX. Update on the extended family of chemokine receptors and introducing a new nomenclature for atypical chemokine receptors. Pharmacol. Rev. 66, 1-79

17 Bonecchi, R. et al. (2008) Regulation of D6 chemokine scavenging activity by ligand- and Rab11-dependent surface up-regulation. Blood 112, 493-503

18 Nibbs, R.J. and Graham, G.J. (2013) Immune regulation by atypical chemokine receptors. Nat. Rev. Immunol. 13, 815-829

19 D'Amico, G. et al. (2000) Uncoupling of inflammatory chemokine receptors by IL-10: generation of functional decoys. Nat. Immunol. $1,387-391$

20 Ariel, A. et al. (2006) Apoptotic neutrophils and T cells sequester chemokines during immune response resolution through modulation of CCR5 expression. Nat. Immunol. 7, 1209-1216

21 Mabuchi, T. et al. (2012) Chemokine receptors in the pathogenesis and therapy of psoriasis. J. Dermatol. Sci. 65, 4-11

22 Mortier, A. et al. (2012) Overview of the mechanisms regulating chemokine activity and availability. Immunol. Lett. 145, 2-9

23 Ebert, L.M. et al. (2005) Chemokine-mediated control of T cell traffic in lymphoid and peripheral tissues. Mol. Immunol. 42, 799-809

24 Tsuchiya, T. et al. (2004) Th1, Th2, and activated T-cell marker and clinical prognosis in peripheral T-cell lymphoma, unspecified: comparison with AILD, ALCL, lymphoblastic lymphoma, and ATLL. Blood 103, 236-241

25 Panina-Bordignon, P. et al. (2001) The C-C chemokine receptors CCR4 and CCR8 identify airway $\mathrm{T}$ cells of allergen-challenged atopic asthmatics. J. Clin. Invest. 107, 1357-1364

26 Sica, A. et al. (1997) Bacterial lipopolysaccharide rapidly inhibits expression of C-C chemokine receptors in human monocytes. J. Exp. Med. 185, 969-974

27 Jeannin, P. et al. (2003) Outer membrane protein A renders dendritic cells and macrophages responsive to CCL21 and triggers dendritic cell migration to secondary lymphoid organs. Eur. J. Immunol. 33, 326-333

28 Forster, R. et al. (2008) CCR7 and its ligands: balancing immunity and tolerance. Nat. Rev. Immunol. 8, 362-371

29 Gouwy, M. et al. (2004) Synergy between proinflammatory ligands of G protein-coupled receptors in neutrophil activation and migration. $J$. Leukoc. Biol. 76, 185-194

30 Gouwy, M. et al. (2008) Synergy between coproduced CC and CXC chemokines in monocyte chemotaxis through receptor-mediated events. Mol. Pharmacol. 74, 485-495

31 Ali, S. et al. (2007) An apparent paradox: chemokine receptor agonists can be used for anti-inflammatory therapy. Mol. Immunol. 44, 1477-1482

32 Hecht, I. et al. (2003) Heterologous desensitization of T cell functions by CCR5 and CXCR4 ligands: inhibition of cellular signaling, adhesion and chemotaxis. Int. Immunol. 15, 29-38 
33 Paoletti, S. et al. (2005) A rich chemokine environment strongly enhances leukocyte migration and activities. Blood 105, 3405-3412

34 Salanga, C.L. et al. (2009) Modulation of chemokine receptor activity through dimerization and crosstalk. Cell. Mol. Life Sci. 66, 1370-1386

35 Sohy, D. et al. (2009) Hetero-oligomerization of CCR2, CCR5, and CXCR4 and the protean effects of "selective" antagonists. J. Biol. Chem. 284, 31270-31279

36 Springael, J.Y. et al. (2006) Allosteric modulation of binding properties between units of chemokine receptor homo- and hetero-oligomers. Mol. Pharmacol. 69, 1652-1661

37 El-Asmar, L. et al. (2005) Evidence for negative binding cooperativity within CCR5-CCR2b heterodimers. Mol. Pharmacol. 67, 460-469

38 Reiter, E. and Lefkowitz, R.J. (2006) GRKs and beta-arrestins: roles in receptor silencing, trafficking and signaling. Trends Endocrinol. Metab. 17, 159-165

39 Raghuwanshi, S.K. et al. (2012) The chemokine receptors CXCR1 and CXCR2 couple to distinct G protein-coupled receptor kinases to mediate and regulate leukocyte functions. J. Immunol. 189, 2824-2832

40 Richardson, R.M. et al. (2003) Role of the cytoplasmic tails of CXCR1 and CXCR2 in mediating leukocyte migration, activation, and regulation. J. Immunol. 170, 2904-2911

41 Lombardi, M.S. et al. (2001) Adjuvant arthritis induces downregulation of $\mathrm{G}$ protein-coupled receptor kinases in the immune system. J. Immunol. 166, 1635-1640

42 Vroon, A. et al. (2006) GRKs and arrestins: regulators of migration and inflammation. J. Leukoc. Biol. 80, 1214-1221

43 Luttrell, L.M. and Kenakin, T.P. (2011) Refining efficacy: allosterism and bias in G protein-coupled receptor signaling. Methods Mol. Biol. $756,3-35$

44 Downes, G.B. and Gautam, N. (1999) The G protein subunit gene families. Genomics 62, 544-552

45 Shenoy, S.K. et al. (2006) beta-arrestin-dependent, G proteinindependent ERK1/2 activation by the beta2 adrenergic receptor. $J$. Biol. Chem. 281, 1261-1273

46 Kenakin, T. and Christopoulos, A. (2013) Signalling bias in new drug discovery: detection, quantification and therapeutic impact. Nat. Rev. Drug Discov. 12, 205-216

47 Rasmussen, S.G. et al. (2011) Crystal structure of the beta2 adrenergic receptor-Gs protein complex. Nature 477, 549-555

48 Rahmeh, R. et al. (2012) Structural insights into biased G proteincoupled receptor signaling revealed by fluorescence spectroscopy. Proc. Natl. Acad. Sci. U.S.A. 109, 6733-6738

49 Dror, R.O. et al. (2011) Activation mechanism of the beta2-adrenergic receptor. Proc. Natl. Acad. Sci. U.S.A. 108, 18684-18689

50 Whalen, E.J. et al. (2011) Therapeutic potential of beta-arrestin- and G protein-biased agonists. Trends Mol. Med. 17, 126-139

51 Tian, Y. et al. (2004) Differential chemokine activation of CC chemokine receptor 1-regulated pathways: ligand selective activation of Galpha 14-coupled pathways. Eur. J. Immunol. 34, 785-795

52 Rajagopal, S. et al. (2013) Biased agonism as a mechanism for differential signaling by chemokine receptors. J. Biol. Chem. 288, 35039-35048

53 Crump, M.P. et al. (1998) Solution structure of eotaxin, a chemokine that selectively recruits eosinophils in allergic inflammation. J. Biol. Chem. 273, 22471-22479

54 Chou, C.C. et al. (2002) Pharmacological characterization of the chemokine receptor, hCCR1 in a stable transfectant and differentiated HL-60 cells: antagonism of hCCR1 activation by MIP1beta. Br. J. Pharmacol. 137, 663-675

55 Berchiche, Y.A. et al. (2011) Different effects of the different natural CC chemokine receptor $2 \mathrm{~b}$ ligands on beta-arrestin recruitment, Galphai signaling, and receptor internalization. Mol. Pharmacol. 79, 488-498

56 O’Boyle, G. et al. (2007) Chemokine-mediated inflammation: Identification of a possible regulatory role for CCR2. Mol. Immunol. 44, 1944-1953

57 Ogilvie, P. et al. (2004) Unusual chemokine receptor antagonism involving a mitogen-activated protein kinase pathway. J. Immunol. $172,6715-6722$

58 Leach, K. et al. (2007) Analysis of second messenger pathways stimulated by different chemokines acting at the chemokine receptor CCR5. Biochem. Pharmacol. 74, 881-890
59 Steen, A. et al. (2013) Biased and constitutive signaling in the CCchemokine receptor CCR5 by manipulating the interface between transmembrane helices 6 and 7. J. Biol. Chem. 288, 12511-12521

60 Oppermann, M. et al. (1999) Differential effects of CC chemokines on CC chemokine receptor 5 (CCR5) phosphorylation and identification of phosphorylation sites on the CCR5 carboxyl terminus. J. Biol. Chem. $274,8875-8885$

61 Byers, M.A. et al. (2008) Arrestin 3 mediates endocytosis of CCR7 following ligation of CCL19 but not CCL21. J. Immunol. 181, 47234732

62 Zidar, D.A. et al. (2009) Selective engagement of G protein coupled receptor kinases (GRKs) encodes distinct functions of biased ligands. Proc. Natl. Acad. Sci. U.S.A. 106, 9649-9654

63 Kohout, T.A. et al. (2004) Differential desensitization, receptor phosphorylation, beta-arrestin recruitment, and ERK1/2 activation by the two endogenous ligands for the CC chemokine receptor 7 . J. Biol. Chem. 279, 23214-23222

64 Watts, A.O. et al. (2012) Label-free impedance responses of endogenous and synthetic chemokine receptor CXCR3 agonists correlate with Giprotein pathway activation. Biochem. Biophys. Res. Commun. 419, $412-418$

65 Wilkie, T.M. et al. (1991) Characterization of G-protein alpha subunits in the Gq class: expression in murine tissues and in stromal and hematopoietic cell lines. Proc. Natl. Acad. Sci. U.S.A. 88, 10049-10053

66 Ahn, S. et al. (2004) Differential kinetic and spatial patterns of betaarrestin and $\mathrm{G}$ protein-mediated ERK activation by the angiotensin II receptor. J. Biol. Chem. 279, 35518-35525

67 Samson, M. et al. (1996) Molecular cloning and functional expression of a new human CC-chemokine receptor gene. Biochemistry 35, 33623367

68 Zabel, B.A. et al. (2009) Elucidation of CXCR7-mediated signaling events and inhibition of CXCR4-mediated tumor cell transendothelial migration by CXCR7 ligands. J. Immunol. 183, 3204-3211

69 Rajagopal, S. et al. (2010) Beta-arrestin- but not G protein-mediated signaling by the "decoy" receptor CXCR7. Proc. Natl. Acad. Sci. U.S.A. 107, 628-632

70 McCulloch, C.V.et al. (2008) Multiple roles for the C-terminal tail of the chemokine scavenger D6. J. Biol. Chem. 283, 7972-7982

71 Odemis, V. et al. (2012) The presumed atypical chemokine receptor CXCR7 signals through $\mathrm{G}(\mathrm{i} / \mathrm{o})$ proteins in primary rodent astrocytes and human glioma cells. Glia 60, 372-381

72 Cardona, A.E. et al. (2008) Scavenging roles of chemokine receptors: chemokine receptor deficiency is associated with increased levels of ligand in circulation and tissues. Blood 112, 256-263

73 Mantovani, A. et al. (2006) Tuning inflammation and immunity by chemokine sequestration: decoys and more. Nat. Rev. Immunol. 6, 907918

74 Schall, T.J. and Proudfoot, A.E. (2011) Overcoming hurdles in developing successful drugs targeting chemokine receptors. Nat. Rev. Immunol. 11, 355-363

75 Lieberman-Blum, S.S. et al. (2008) Maraviroc: a CCR5-receptor antagonist for the treatment of HIV-1 infection. Clin. Ther. 30, $1228-1250$

76 De Clercq, E. (2010) Recent advances on the use of the CXCR4 antagonist plerixafor (AMD3100, Mozobil) and potential of other CXCR4 antagonists as stem cell mobilizers. Pharmacol. Ther. 128, $509-518$

77 Soergel, D.G. et al. (2014) First clinical experience with TRV130: Pharmacokinetics and pharmacodynamics in healthy volunteers. $J$. Clin. Pharmacol. 54, 351-357

78 DeWire, S.M. et al. (2013) A G protein-biased ligand at the mu-opioid receptor is potently analgesic with reduced gastrointestinal and respiratory dysfunction compared with morphine. J. Pharmacol. Exp. Ther. 344, 708-717

79 Pierce, K.L. et al. (2002) Seven-transmembrane receptors. Nat. Rev. Mol. Cell Biol. 3, 639-650

80 Venkatakrishnan, A.J. et al. (2013) Molecular signatures of G-proteincoupled receptors. Nature 494, 185-194

81 Preininger, A.M. et al. (2013) Conformational flexibility and structural dynamics in GPCR-mediated G protein activation: a perspective. $J$. Mol. Biol. 425, 2288-2298 
82 Ritter, S.L. and Hall, R.A. (2009) Fine-tuning of GPCR activity by receptor-interacting proteins. Nat. Rev. Mol. Cell Biol. 10, 819-830

83 Alexander, S.P. et al. (2011) Guide to Receptors and Channels (GRAC), 5th edition. Br. J. Pharmacol. 164 (Suppl 1), S1-S324

84 Jensen, P.C. et al. (2008) Positive versus negative modulation of different endogenous chemokines for CC-chemokine receptor 1 by small molecule agonists through allosteric versus orthosteric binding. J. Biol. Chem. 283, 23121-23128

85 Tchernychev, B. et al. (2010) Discovery of a CXCR4 agonist pepducin that mobilizes bone marrow hematopoietic cells. Proc. Natl. Acad. Sci. U.S.A. 107, 22255-22259
86 Quoyer, J. et al. (2013) Pepducin targeting the C-X-C chemokine receptor type 4 acts as a biased agonist favoring activation of the inhibitory G protein. Proc. Natl. Acad. Sci. U.S.A. 110, 5088-5097

87 Saita, Y. et al. (2006) Structural basis for the interaction of CCR5 with a small molecule, functionally selective CCR5 agonist. J. Immunol. 177, 3116-3122

88 Ferain, T. et al. (2011) Agonist-induced internalization of CC chemokine receptor 5 as a mechanism to inhibit HIV replication. $J$. Pharmacol. Exp. Ther. 337, 655-662 